\title{
On a Hybrid Beamforming/Space-Time Coding Scheme
}

\author{
Li Ping, Member, IEEE, Linrang Zhang, Member, IEEE, and H. C. So, Member, IEEE
}

\begin{abstract}
A multiple transmit antenna system based on hybrid beamforming and space-time coding technologies is examined. The reduction factor of the required transmitted energy achievable by the use of hybrid scheme is quantified for any given outage capacity. We show that although a sole space-time coding configuration is superior asymptotically (i.e., for extremely low outage requirements), a hybrid beamforming/space-time coding configuration can be a more effective solution for modest outage requirements. It may provide a useful design guideline for wireless systems, especially for the downlink where multiple transmit antenna scheme is feasible.
\end{abstract}

Index Terms-Beamforming, smart antennas, space-time coding, transmit diversity.

\section{INTRODUCTION}

B EAMFORMING and space-time coding [1]-[3] represent two different approaches to enhance the performance of wireless systems employing multiple transmit antennas. It is reported in [3] that with a four-transmit-antenna setting in quasistatic fading environment, space-time coding is asymptotically superior to beamforming. The comparison in [3] is based on specific forward error correction (FEC) coding and space-time coding scheme. The concept of hybrid beamforming and diversity has also been studied [4], [5] and its advantages have been demonstrated using pair-wise error probability [4] and specific space-time codes [4], [5].

In this letter, we study the hybrid beamforming and diversity scheme from outage capacity point of view. We will quantify the reduction factor of the required transmitted energy achievable by the use of hybrid scheme for any given outage capacity. We will show that although a sole space-time coding configuration is superior asymptotically (i.e., for extremely low outage requirements), a hybrid beamforming/space-time coding configuration can be a more effective solution for modest outage requirements, which may provide a useful design guideline for wireless systems, especially for the downlink.

Manuscript received April 15, 2003. The associate editor coordinating the review of this letter and approving it for publication was Prof. A. Annamalai.This work was fully supported by a strategic grant from City University of Hong Kong, Hong Kong, under Project 7001383.

L. Ping is with the Department of Electronic Engineering, City University of Hong Kong, Kowloon, Hong Kong (e-mail: eeliping@ @ityu.edu.hk).

L. Zhang is with the Key Lab of Radar Signal Processing, Xidian University, Xi'an 710071, China (e-mail: lrzhang@xidian.edu.cn).

H. C. So is with the Department of Computer Engineering \& Information Technology, City University of Hong Kong, Kowloon, Hong Kong (e-mail ithcso@cityu.edu.hk).

Digital Object Identifier 10.1109/LCOMM.2003.822520

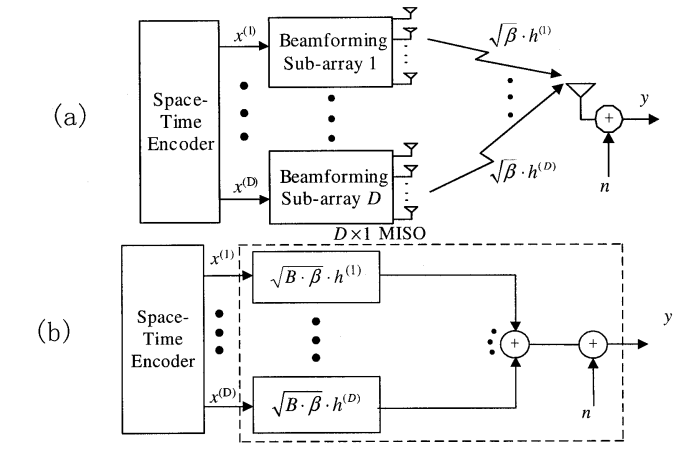

Fig. 1. (a) The generic system. (b) The equivalent system based on a MISO channel model.

\section{SySTEM MODEL}

Following [4], the structure of a hybrid beamforming and diversity scheme is depicted in Fig. 1(a). We assume that $N=$ $D \times B$ transmit antennas are used. These $N$ antennas are divided into $D$ sub-arrays, each consisting of $B$ antennas. Such a scenario is denoted as a $(D, B)$ configuration hereafter. Our aim is to identify the optimal configuration $(D, B)$. To simplify the analysis, some assumptions are listed as follows.

1) Only one receive antenna is used.

2) Half wavelength spacing is used for antennas within each sub-array. Similar to [4], we assume that there is a single path from the mobile to each sub-array and the channels seen by the antennas in a given sub-array are perfectly correlated which can be described by an array response vector $\boldsymbol{a}_{i}(i=1,2, \ldots, D)$. We can estimate $\boldsymbol{a}_{i}(i=1,2, \ldots, D)$ through feedback or other direction-of-arrival (DOA) estimation techniques. The corresponding normalized array response vector $(1 / \sqrt{B}) \cdot \boldsymbol{a}_{i}$ is directly used as the beamforming weight vector for the $i$ th sub-array. Perfect beamforming is assumed for each sub-array, which achieves the maximum aperture gain equal to $B$. (Aperture gain can be viewed as an increase in the mean of the signal power at the desired direction achieved by an antenna array relative to one antenna, assuming the same total transmitted power for both systems [5].)

3) The sub-arrays are separated far enough to ensure that signal from each transmit sub-array experiences independent fading. We treat each sub-array as an equivalent transmit unit. Space-time coding is then applied to the $D$ equivalent units to achieve transmit diversity.

4) $h^{(i)}(i=1,2, \ldots, D)$ is the fast fading coefficient from the $i$ th transmit sub-array to the receive antenna, modeled as an independent zero-mean complex Gaussian random 
variable with unity variance, and $\beta$ is the shadow fading and path loss that, for simplicity, is assumed common for signals from all the sub-arrays to the receive antenna.

5) We restrict our attention to quasi-static flat Rayleigh fading channels.

6) The system is fully synchronous.

Notice that $(1, N)$ and $(N, 1)$ configurations represent, respectively, a sole beamforming and a sole space-time coding system.

The system can be described by an equivalent $D \times 1$ multiple-input-single-output (MISO) system in Fig. 1(b). When the channel coefficients $\left\{h^{(i)}\right\}$ are fixed and unknown to the transmitter, the capacity of the system in Fig. 1(b) is [1]

$$
C=\log _{2}(1+\gamma)
$$

where $\gamma$ is defined by

$$
\gamma \equiv\left(\frac{B}{D}\right) \cdot\left(\frac{E_{t}}{N_{0}}\right) \cdot \beta \cdot \sum_{i=1}^{D}\left|h^{(i)}\right|^{2}
$$

with $E_{t}$ the total average transmitted energy per symbol and $N_{0}$ the variance of the additive white noise at the receiver.

Assuming that $x^{(1)}, \ldots, x^{(D)}$ are independent random variables and $E\left(\left|x^{(i)}\right|^{2}\right)=E_{t} / D, \forall_{i}$, then $\gamma$ is the average signal-to-noise ratio (SNR) averaged over the distribution of $\left\{x^{(i)}\right\}$ at the receiver.

\section{Performance Analysis}

Since $\left\{h^{(i)}\right\}$ are random variables, so are $\gamma$ and $C$. Given a specified transmission rate $\hat{C}$, we can calculate the corresponding $\hat{\gamma}$ from (1), i.e., $\hat{C}=\log _{2}(1+\hat{\gamma})$. Then $\operatorname{Pr}(C \leq \hat{C})=$ $\operatorname{Pr}(\gamma \leq \hat{\gamma})$ is defined as the outage capacity [1]. Outage capacity provides the theoretical limit of transmission failure caused by fading, assuming ideal beamforming within each sub-array and ideal space-time coding among $D$ sub-arrays. In the following, we will use outage capacity as a performance criterion.

Since $\left\{h^{(i)}\right\}$ are complex Gaussian random variables, $\sum_{i=1}^{D}\left|h^{(i)}\right|^{2}=\sum_{i=1}^{D}\left(\left|\operatorname{Re}\left(h^{(i)}\right)\right|^{2}+\left|\operatorname{Im}\left(h^{(i)}\right)\right|^{2}\right)$ is a chi-squared random variable with $2 D$ degrees of freedom [1]. The probability density function of $\gamma$ is

$$
\begin{gathered}
p_{\gamma}(\gamma)=\frac{1}{\left(\frac{B}{D}\right) \cdot\left(\frac{E_{t}}{N_{0}}\right) \cdot \beta \cdot(D-1) !} \cdot\left[\frac{\gamma}{\left(\frac{B}{D}\right) \cdot\left(\frac{E_{t}}{N_{0}}\right) \cdot \beta}\right]^{D-1} \\
\cdot \exp \left(-\frac{\gamma}{\left(\frac{B}{D}\right) \cdot\left(\frac{E_{t}}{N_{0}}\right) \cdot \beta}\right)
\end{gathered}
$$

The outage capacity $P_{o}$ is computed as

$$
\begin{aligned}
P_{o} & =\operatorname{Pr}(\gamma \leq \hat{\gamma}) \\
& =\int_{0}^{\hat{\gamma}} p_{\gamma}(\gamma) d \gamma \\
& =1-\left\{\sum_{d=0}^{D-1} \frac{1}{d !} \cdot\left[\frac{\hat{\gamma}}{\left(\frac{B}{D}\right) \cdot\left(\frac{E_{t}}{N_{0}}\right) \cdot \beta}\right]^{d}\right\}
\end{aligned}
$$

$$
\begin{aligned}
& \cdot \exp \left(-\frac{\hat{\gamma}}{\left(\frac{B}{D}\right) \cdot\left(\frac{E_{t}}{N_{0}}\right) \cdot \beta}\right) \\
= & \left\{\sum_{d=D}^{\infty} \frac{1}{d !} \cdot\left[\frac{\hat{\gamma}}{\left(\frac{B}{D}\right) \cdot\left(\frac{E_{t}}{N_{0}}\right) \cdot \beta}\right]^{d}\right\} \\
& \cdot \exp \left(-\frac{\hat{\gamma}}{\left(\frac{B}{D}\right) \cdot\left(\frac{E_{t}}{N_{0}}\right) \cdot \beta}\right)
\end{aligned}
$$

We write $P_{o}$ as a function of $\hat{\gamma}, \beta$, and $E_{t} / N_{0}$ indexed on $D$ and $B$

$$
P_{o}=f_{D, B}\left(\frac{\hat{\gamma}}{\beta \cdot \frac{E_{t}}{N_{0}}}\right) .
$$

Given the outage capacity $P_{o}$ and fixing $(D, B)$, the required transmitted energy is

$$
\left(E_{t}\right)_{D, B}=\frac{\hat{\gamma} \cdot N_{0}}{f_{D, B}^{-1}\left(P_{o}\right) \cdot \beta} .
$$

We define the required energy reduction factor (RERF) as

$$
\begin{aligned}
\mathrm{RERF} & \equiv 10 \log _{10}\left(E_{t}\right)_{1,1}-10 \log _{10}\left(E_{t}\right)_{D, B} \\
& =10 \log _{10} \frac{f_{D, B}{ }^{-1}\left(P_{o}\right)}{f_{1,1}{ }^{-1}\left(P_{o}\right)}
\end{aligned}
$$

which represents the difference between the required transmitted energy levels for $(1,1)$ and $(D, B)$ configurations, assuming that $\hat{\gamma}, \beta$, and $N_{0}$ remain the same for both cases. (Note: The $(1,1)$ configuration is simply a single transmit antenna system.) Notice that RERF is a function of $P_{o}$ and is independent of $\hat{\gamma}, N_{0}$ and $\beta$.

For extremely low outage requirements, the following discussion indicates that a sole space-time coding configuration is always superior. Denote $\xi=\hat{\gamma} /\left(N \cdot\left(E_{t} / N_{0}\right) \cdot \beta\right)$ and consider $P_{o} \rightarrow 0$. In this case, from (6), it can be shown that $E_{t} \rightarrow \infty$ and so $\xi \cdot D^{2} \rightarrow 0$. Then we can approximate (4) as

$$
\left(P_{o}\right)_{D} \approx \frac{1}{D !} \cdot\left(\xi \cdot D^{2}\right)^{D} \cdot \exp \left(-\xi \cdot D^{2}\right) .
$$

For a given $N$, the basic relationship (4) (and so (8)) is not defined for all $D$, since we require that $D$ should divide $N$. However, we treat (8) as a nominal expression. We now show that $P_{o}$ is monotonously decreasing when $D$ increases (for $E_{t} \rightarrow \infty$ ). From (8)

$$
\frac{\left(P_{o}\right)_{D}}{\left(P_{o}\right)_{D+1}} \approx\left(\frac{D}{D+1}\right)^{2 D} \cdot \frac{1}{\xi \cdot(D+1)} \cdot \exp [\xi \cdot(2 D+1)] .
$$

For sufficiently small $\xi \exp [\xi \cdot(2 D+1)]>1$ and $(1 /(\xi \cdot$ $(D+1)))>e^{2}$. It can also be shown that $\left(1 / e^{2}\right)<(D /(D+$ 1) $)^{2 D}<1$ for $D \geq 1$. Substituting these into (9), we obtain

$$
\frac{\left(P_{o}\right)_{D}}{\left(P_{o}\right)_{D+1}}>1, \quad \text { for } E_{t} \rightarrow \infty \text {. }
$$

Thus the $(N, 1)$ configuration (i.e., the sole space-time coding case) is advantageous asymptotically. However, for a finite 


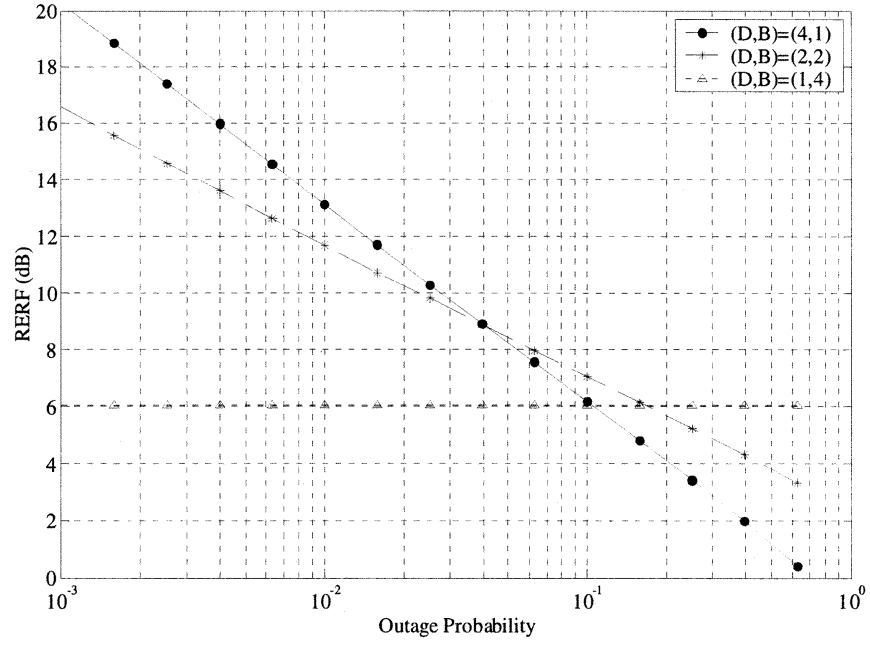

Fig. 2. RERF versus outage probability for four-transmit-antenna systems.

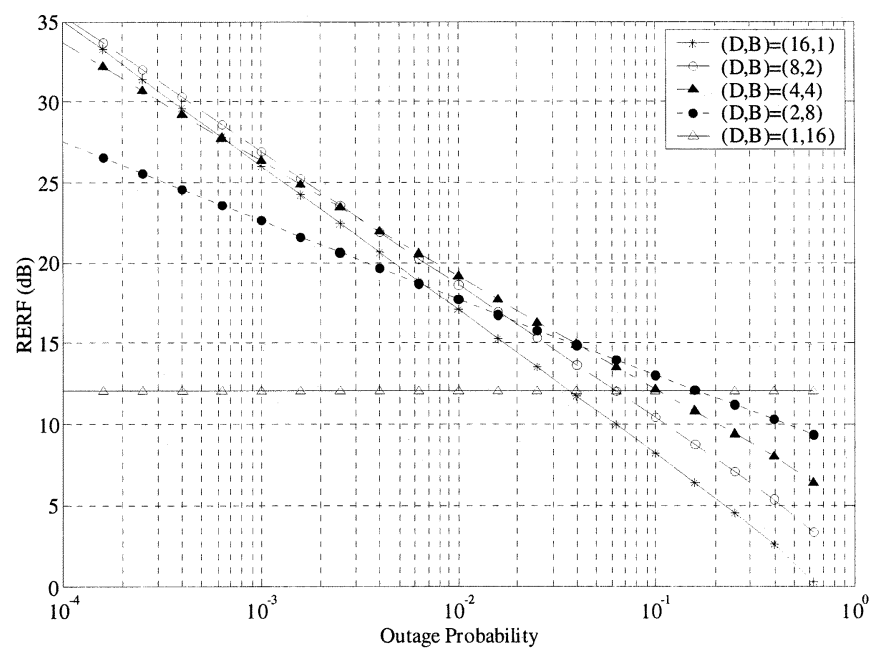

Fig. 3. RERF versus outage probability for sixteen-transmit-antenna systems

$P_{o}$ requirement, other configurations can be better choices, as shown below.

\section{NUMERICAL RESULTS}

Fig. 2 shows RERF versus $P_{o}$ for four-transmit-antenna systems. Considering a common outage requirement of
$P_{o}=10^{-2}$, the RERF values are approximately $13.1,11.7$, and $6 \mathrm{~dB}$ for $(D, B)=(4,1),(D, B)=(2,2)$ and $(D, B)=(1,4)$ systems, respectively. This means that, e.g., a $(4,1)$ system can save $13.1 \mathrm{~dB}$ of transmitted energy compared with a (1, 1) reference system. Space-time coding is the best choice in this case, which is consistent with the result in [3]. However, for $P_{o}>4 \times 10^{-2}$, other configurations can be better choices. This is demonstrated more clearly in Fig. 3 for various $D \times B=16$ configurations. For $P_{o}=10^{-2}$, the best choice is $(D, B)=(4,4)$ which has about 2 -dB improvement over the sole space-time coding $(16,1)$ configuration.

\section{CONCLUSIONS}

In a cellular environment, transmitted energy from one user will become interference to others. Any reduction of the transmitted energy can be translated into a capacity gain and so is highly desirable. In this letter, we have investigated the reduction of required transmitted energy using multiple transmit antenna techniques and we have shown the advantage of hybrid beamforming/space-time coding configurations. This may provide useful information for multiple antenna system design.

Other advantages of hybrid systems are related to sensitivity and complexity. When the number of transmit antennas is large, a sole beamforming configuration is very sensitive to the error in DOA estimation while a sole space-time coding configuration may require a high receiver complexity. A hybrid system may avoid the difficulties in these two extreme situations.

\section{REFERENCES}

[1] G. J. Foschini and M. J. Gans, "On limits of wireless communication in a fading environment when using multiple antennas," Wireless Pers. Commun., vol. 6, no. 3, pp. 311-335, Mar. 1998.

[2] A. Narula, M. D. Trott, and G. W. Wornell, "Performance limits of coded diversity methods for transmitter antenna arrays," IEEE Trans. Inform. Theory, vol. 45, pp. 2418-2433, Nov. 1999.

[3] G. Bauch and J. Hagenauer, "Smart versus dumb antennas-capacities and FEC performance," IEEE Commun. Lett., vol. 6, pp. 55-57, Feb. 2002.

[4] R. W. Heath Jr. and A. Paulraj, "Multiple antenna arrays for transmitter diversity and space-time coding," in Proc. IEEE Int.Conf. on Communications (ICC'99), vol. 1, June 1999, pp. 36-40.

[5] R. A. Soni, R. M. Buehrer, and R. D. Benning, "Intelligent antenna system for cdma2000," IEEE Signal Processing Mag., vol. 19, no. 4, pp. 54-67, July 2002. 